\title{
On the spatial distribution of the galactic Wolf-Rayet stars
}

\author{
Emilio J. Alfaro ${ }^{1}$, Miguel Cerviño ${ }^{2}$, J.A. Rubiño ${ }^{1,4}$, \\ Jesus Cabrera-Caño ${ }^{1,3}$, and José M. Vílchez ${ }^{1,4}$ \\ 1 IAA (CSIC), P.O. Box 3004, E-18008 Granada, Spain \\ 2 LAEFF (INTA), P.O. Box 50727, E-28080 Madrid, Spain \\ 3 Universidad de Sevilla, P.O. Box 1045, E-18080 Sevilla, Spain \\ 4 IAC, c/ Vía Láctea $s / n$, E-38200 La Laguna, Tenerife, Spain
}

\begin{abstract}
From a new compilation of spectral types and photometry (version April '98 on the Web) performed by van der Hucht for the Galactic WR stars, we have estimated the distance using two different luminosity-spectral type calibrations. Both determinations lead to similar results. On the basis of newly determined distances we have performed a quantitative analysis of the spatial density distribution for the WN and WC spectral types as well as for the whole WR sample and compared them to the one depicted by the large star-forming regions delineated by the population of young open clusters (YOCs). WR stars show a clumpy distribution similar to the one shown by the YOCs and the WN and WC spectral classes present different galactic distributions as well. Otherwise, variations of the WC/WN quotient do not show a clear correlation with the galactic metallicity gradient, on the contrary this ratio seems to be constant on regions of about $1 \mathrm{kpc}$ in size and shows significant variations (even by a factor of 8 ) between the closest star-forming complexes.
\end{abstract}

\section{Results and discussion}

We have used two different $M_{v}$-spectral type relationships to estimate the distance of the cataloged galactic WR stars: the first one has been taken from van der Hucht (1995) and the second one has been derived by Cerviño et al. (in preparation) and is based on the three-dimensional spectral classification for WN stars by Smith et al. (1996).

Density-distribution maps for the WR stars and, separately, for the WC and WN spectral types have been obtained via Kernel estimators following the methodology described in Alfaro et al. (1992). This analysis is limited to a volume with $r=4 \mathrm{kpc}$ centered around the Sun, where the WR sample is supposed to be complete. WR stars show a clumpy distribution outlining three of the main star-forming regions drawn by optical tracers (Alfaro et al. 1992), but two complexes placed outside the solar circle do not show WR counterparts. This result adds new support to metallicity as being one of the main factors controlling the evolution of massive O-type stars to WR stages.

In the same way we have obtained the WC/WN ratio map shown in Fig. 1. The smoothing parameters chosen to evaluate the Kernel density distributions have been $\mathrm{hx}=\mathrm{hy}=0.4 \mathrm{kpc}$ in order of increasing the signal to noise ratio in each point at the expense of increasing the bias too (see Silverman 1986). The 

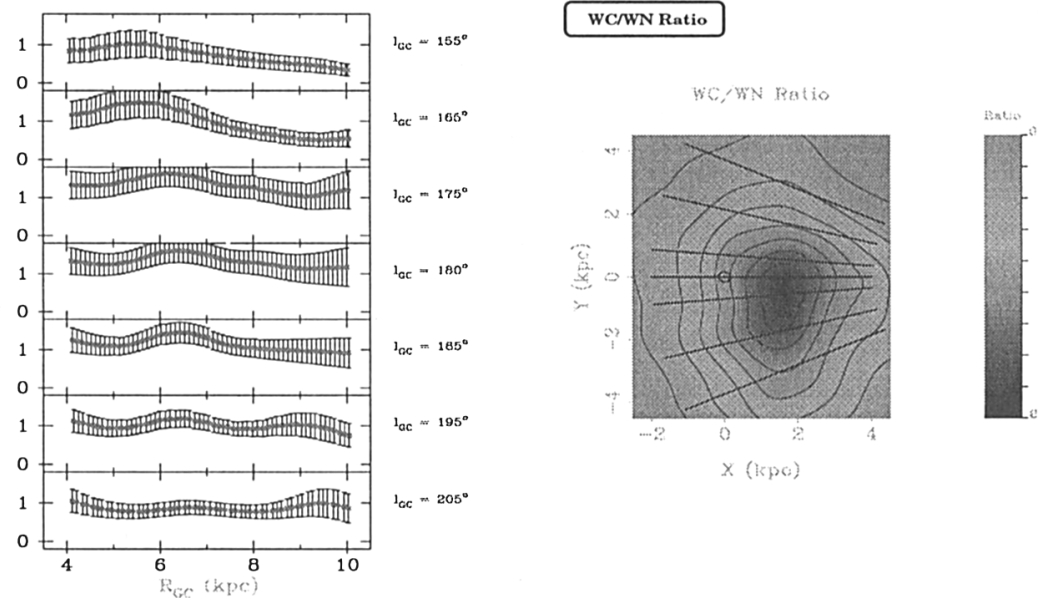

Figure 1. WC/WN ratio. The sun is placed in $(0,0)$ in the right panel.

results show that the maximum $\mathrm{WC} / \mathrm{WN}$ value is found in the Sagittarius region and reaches up to $1.8 \pm 0.2$. Fig. 1 demonstrates that the solar vicinity does not show a homogeneous WC/WN distribution, but varies between 1.8 and 0.2 in short spatial scales and, overall, that metallicity is not the main factor driving the $\mathrm{WC} / \mathrm{WN}$ ratio in the solar neighborhood (note that to get a WC/WN ratio of 1.8 , most of the models predict metallicities lower than one hundredth of solar one).

The star-forming super-complexes represent large $(\sim 1 \mathrm{kpc})$ regions of coherent star formation where the age spread scales with a power law of size. The variations of the $\mathrm{WC} / \mathrm{WN}$ ratio found for the three different WR clumps detected in the solar vicinity could be, in this way, interpreted as a function of age. Leitherer (these Proceedings) discusses the role played by age in different evolutionary models, and suggests that a high $\mathrm{WC} / \mathrm{WN}$ ratio $(>0.5)$ can only be obtained for a narrow range of age assuming a solar metallicity (however, see Mas-Hesse \& Cerviño, these Proceedings).

\section{References}

Alfaro, E.J., Delgado, A., Cabrera-Caño, J. 1992, ApJ 386, L47

Silverman, B.W. 1986, Density Estimation for Statistic and Data Analysis (London: Chapma and Hall)

Smith, L.F., Shara, M.M., Moffat, A.F.J. 1996, MNRAS 281, 163

van der Hucht, K.A 1995, in: K.A. van der Hucht \& P.M. Williams (eds.), Wolf-Rayet Stars, Binaries, Colliding Winds, Evolution, Proc. IAU Symp. No. 163 (Dordrecht: Kluwer), p. 7 